\title{
Curvature flows and barriers in fire front modelling
}

\author{
$\underline{\text { V.-M. Wheeler }}^{\text {a }}$, J. A. McCoy ${ }^{\text {a }}$, G. E. Wheeler ${ }^{\text {a }}$, J. J. Sharples ${ }^{\text {b }}$ \\ ${ }^{a}$ Institute for Mathematics and its Applications, School of Mathematics and Applied Statistics, Faculty of \\ Informatics, University of Wollongong, Wollongong, NSW 2522, Australia \\ ${ }^{\mathrm{b}}$ Applied and Industrial Mathematics Research Group, School of Physical, Environmental and Mathematical \\ Sciences, University of New South Wales Canberra, PO Box 7916, Canberra BC 2610, Australia \\ Email: vwheeler@uow.edu.au
}

\begin{abstract}
In this paper we propose a novel mathematical model for describing the evolution of a fire front. Specifically, for a homogeneous fuel bed of varying height with constant ignition temperature $T_{i g}$ we model the isosurface corresponding to $T_{i g}$. The intersection of this isosurface with the fuel bed defines the evolving front.

There are three natural processes driving the evolution of the evolving front: radiative heat transfer, convective heat transfer, and heat/mass transfer. These processes clearly depend on the shape of the isosurface in space, and this nonlinear feedback can be understood through the curvature of the isosurface. The model we propose here is a second-order nonlinear evolution equation, where the movement of the front depends on the curvature of the two-dimensional isosurface in three dimensional space in addition to atmospheric, topographic and fuel conditions.
\end{abstract}

This approach has marked advantages over traditional linear evolution equations of curves, such as those arising from Huygen's principle. Vertical variations in fuel and weather can be more realistically incorporated, as can barriers blocking the progress of the fire. Our second-order model is also more accurate. Evidence to support this comes in the form of previous experimental fire modelling results, which showed that initial corners of a fire front become immediately smooth. Such behaviour cannot be obtained using a quasi-steady speed of evolution, but is, however, consistent with the presence of a curvature term in the speed.

Modelling the fire front as a surface in space rather than a curve in the plane allows us to better incorporate topography and fuel variations and to examine flame height as a fire passes through an urban or rural region. Further, our introduction of free boundaries for the evolving surface is a new approach in the modelling of fire fronts with barriers. The future behaviour of an existing fire could be understood by predicting the shape of the front using our model, but more significantly, the evolving fire front could be controlled and even forced to extintion in a finite time, by constructing an appropriate back burn region or regions that act as free boundaries in the mathematical model.

Our foci in this paper are to describe the new model, compare it with previous approaches to modelling fire front propagation and present a couple of simplified cases that have already been studied from the analytical point of view. We also outline how improvements introduced to the analytical model will allow a better understanding and prediction of fire fronts.

Keywords: Curvature flow, partial differential equation, free boundary problem, fire front, barrier 


\section{INTRODUCTION}

Recently there have been exciting developments in the theory of geometric evolution equations, which govern the deformation of curves and surfaces, and their application to diverse problems within mathematics, in physics and beyond. One of the most studied deformations of surfaces is the mean curvature flow. Some of the latest developments concern improved understanding of mean curvature flow of surfaces with free boundaries and related flows which incorporate a source term into the evolution. Combining these recent ideas yields an exciting new approach to modelling the propagation and long time behaviour of fire fronts; in this article we describe the idea and the existing results that are particularly relevant to the application.

\subsection{Background on modelling fire fronts}

The propagation and long time behaviour of a fire front is of widespread interest to scientists, geographers, historians and mankind generally. Fire, when uncontrolled, is one of the most destructive natural forces, with evidence of catastrophic mass fires in Australia dating back as far as 350 million years. A key motivation for fire front and bushfire research is to give a better understanding of the development and likely impacts of an already-formed fire or bushfire. This in turn allows for fire fighting techniques to be improved, helping to prevent loss of life and material assets.

Presently the literature lacks a common model which describes the evolving fire front. One difficulty is to fit the model into a unified set of equations at each of the micro-, meso-, macro- and gigascopic-scales (SéroGuillaume et al., 2007). The three types of model accepted so far are the physical, the semi-physical, and the empirical (Perry, 1998). Physical models are rigorous mathematical formulations based upon the chemical and physical phenomena underlying fire expansion; empirical models are statistical in nature, mainly concerned with fire shape prediction; and the semi-physical or semi-empirical models are a combination of both. The last approach predicts the shape of the fire front while also taking into consideration the underlying conditions of terrain and atmospheric changes. Several authors have suggested that fire fronts evolve with a speed depending on the curvature of the interface as well as atmospheric and topographic parameters (Osher and Sethian, 1988; Roberts, 1993; Sapiro and Tannenbaum, 1994; Weber and Sidhu, 2006). Nevertheless, due to the difficulties that the curvature term brings, most existing results are obtained under the assumption that the large scale of the fire permits the speed to be reasonably approximated by ignoring the curvature term (Anderson et al., 1982; Roberts, 1993; Séro-Guillaume et al., 2007). In this vein, we mention the numerical studies based on the Hamilton-Jacobi equations of a developing fire front (Osher and Sethian, 1988) and the equation of the fire length with or without suppression terms (Weber and Sidhu, 2006). The main method used to predict the shape of a wildland fire is Huygens principle, as proposed for this application by Anderson et al. (1982); this method approximates the fire front at each future time by a locally-defined ellipse starting on the previous time line. Numerical results based on a simplified empirical model of fire front evolution depending on the wind velocity have been obtained by Mallet et al. (2009). Optimal blocking problems in the plane have been used to predict the cost of construction of a barrier to a forest fire, (Bressan and Wang, 2012, and the references therein). In these cases the evolving fire front is assumed to have a speed depending linearly on the position vector.

\subsection{Background on curvature flow}

A normal flow is the time evolution of a manifold, be it a curve, a surface or a higher-dimensional "hypersurface", where each individual point moves in the normal direction to the surface there with a prescribed speed. Depending on the problem to be modeled, the speed may be chosen such that the flow minimises a certain functional defined on the hypersurface (as for example surface area or volume). Then the flow is the steepest descent gradient flow of the chosen functional. Often the speed is dependent on geometric quantities of the evolving surface, such as its curvature, and possibly derivatives of curvature. These quantities can be represented in terms of the chosen parametrisation of the hypersurface and its derivatives. Depending on the speed, the flow can be first-order (no derivatives of the parametrisation are involved in the speed), second-order as mean curvature flow (curvatures are involved), or higher-order (derivatives of curvature involved). Normal flows have a vast application to the modelling of physical phenomenons, from grain boundaries, annealing metals, moving interfaces, image processing, biological membranes and many more. An evolution speed depending on curvature gives rise to a system of second-order parabolic partial differential equations describing the evolving surface, in this application a fire front.

Perhaps the most well known curvature flow is the mean curvature flow, which was introduced in the 1950s for modelling the motion of grain boundaries in recrystallised metals such as copper (Mullins, 1956). 
Mean curvature flow was first studied by methods of classical analysis of partial differential equations by Huisken (1984). He showed that smooth, convex hypersurfaces converge in a finite maximal time to a point, which, under a suitable rescaling, approaches exponentially a sphere. This work spawned research into curvature flow of hypersurfaces in many directions, including mean curvature flow with a constraint term in several settings (Andrews, 2001; McCoy, 2005, and the references therein). Further, there has also been progress on mean curvature flow with free boundary (Stahl, 1996; Wheeler (nee Vulcanov), 2012, 2013, and the references therein), where the flow is constrained to a certain domain with boundary depending on time.

\section{CURVATURE DEPENDENT MODEL FOR FIRE FRONT EVOLUTION}

Up until now, there has been no complete analytical study of a model that can accurately represent the evolution of a fire front. Experiments showed that the corner of an initially triangular fire front will smooth out almost instantaneously and the fire front will develop into a roughly ellipsoidal shape after some time (Anderson et al., 1982). This behaviour cannot be obtained if the curvature term in the evolution is neglected. It is instead consistent with the presence of a mean curvature term, or indeed a fully nonlinear curvature flow such as one of those identified by Andrews et al. (2013) with the instantaneous smoothing property. Although the model proposed here based on the mean curvature flow is significantly more complicated than those used classically, it will model much more accurately the behaviour of the fronts since it does not neglect the curvature term. Further, such a model has the concept of barriers for the fire front built-in, allowing one to apply theoretical knowledge to evaluate the effects of different control options on the behaviour of a developing fire.

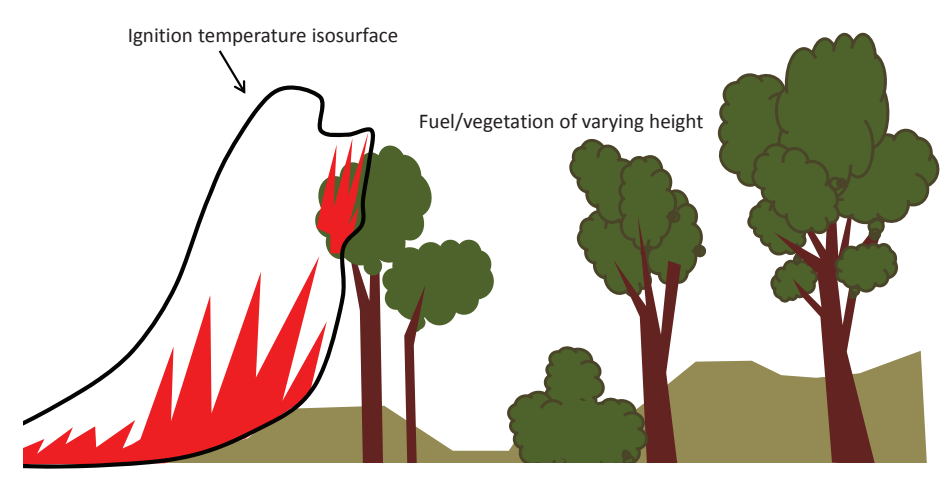

Figure 1. Schematic illustrating the ignition temperature isosurface and how it relates to fuel of varying type and height.

Until now fire fronts have been modelled as curves evolving in the plane, corresponding to partial differential equations with one spatial variable. In reality, a fire front is a two-dimensional interface between a threedimensional unburnt region (the fuel) and a three-dimensional burnt region. These three dimensional regions are not homogeneous in general; they could be rural regions of grassland, vegetation of varying height or urban regions including buildings (some perhaps containing particularly flammable materials), roads, other structures and so forth. There may also be further obstacles such as areas of water or desert regions which can influence an evolving fire front.

Here we propose to model the isosurface of ignition temperature for a homogenous fuel region with an existing barrier. In essence this approach can be viewed as a generalisation of the approach adopted by Rothermel (1972) to model surface fire spread. Mathematically, this translates to the time evolution of a surface in threedimensional space, with normal velocity equal to a function depending on the mean curvature of the surface and an extra source function. At each point $(p, t) \in M^{2} \times[0, T)$, we require:

$$
\frac{\partial F}{\partial t} \cdot \nu_{M_{t}}=-H+f
$$

Here $M^{2}$ is the fixed parameter manifold with boundary denoted by $\partial M^{2}$ where the superscript 2 indicates the dimension of the manifold. The time interval $[0, T)$ is to be determined, when (1) is provided with suitable initial and boundary conditions. Above we have denoted by $M_{t}=F(\cdot, t)$ the evolving isosurface of the ignition temperature at time $t$, by $\nu_{M_{t}}$ the unit normal to $M_{t}$ and by $H$ the mean curvature of $M_{t}$. By $f$ 
we denote an explicitly given function determined from experimental data, and depending on the direction of the wind $n_{w}$, combustion properties of the fuel and topographical information. The initial surface is $M_{0}=$ $F_{0}\left(M^{2}\right)$, a two dimensional surface with boundary formed from one or more disconnected components. The boundary is defined as $\partial M_{0}=F_{0}\left(\partial M^{2}\right)=\Sigma \cap M_{0}$. Here we have introduced another fixed surface $\Sigma$, the support surface, which contains the moving boundary of $M_{t} . \Sigma$ can also be seen as a reunion of disconnected surfaces if the parameter manifold has more than one boundary components. Each of the support surfaces which are contained in $\Sigma$ corresponds to a barrier or obstacle (consisting of geographical barriers such as rivers or roads, or a back-burn, as examples) blocking the passage of the fire front. To our knowledge such a barrier consideration has only very recently been considered theoretically in connection with mean curvature flow and with no source function $f$ (Almeida et al., 2012). For example the simplest case is the case of a surface moving between two planes, one at height zero and another at a constant positive height, with two disconnected boundary curves one contained in each of the two planes.

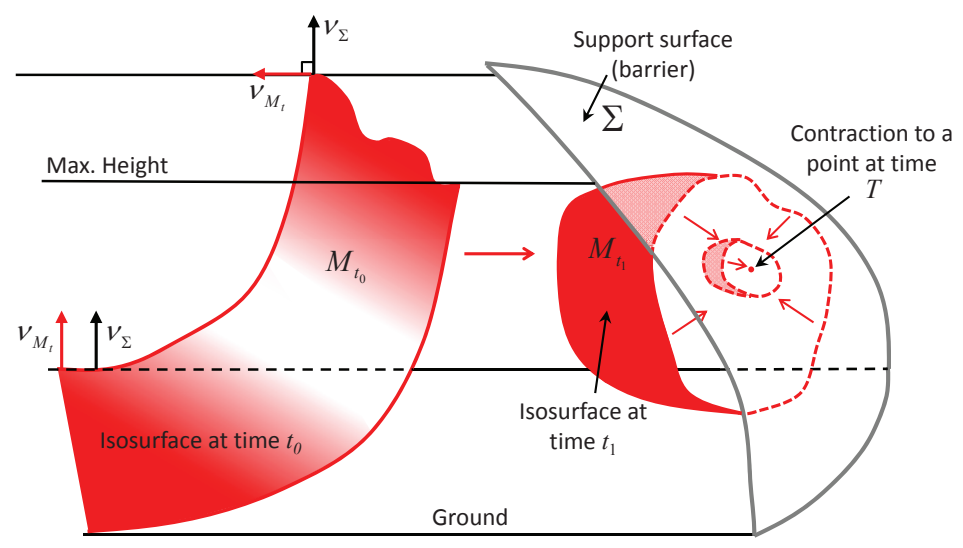

Figure 2. Schematic illustrating the evolution of the surface $M_{t}$ in relation to a support surface $\Sigma$. The diagram also illustrates the surface $M_{t}$ contracting to a point on $\Sigma$ as $t \rightarrow T$.

Depending on the scale at which the problem is considered, the boundary conditions for the evolution will be either:

1. The normal of the evolving fire front, $\nu_{M_{t}}$, and the normal of the barrier $\Sigma, \nu_{\Sigma}(F)$, are parallel; that is,

$$
\nu_{M_{t}} \cdot \nu_{\Sigma}(F)=1
$$

Note that the normal to $\Sigma$ is evaluated at points along the evolving surface, that is $\nu_{\Sigma}(F)=\nu_{\Sigma} \circ F$. The boundary condition (2) corresponds to a zoomed-in look at the boundary behaviour, where the fire would be seen as "creeping" up the barrier.

2. The normal of the evolving fire front, $\nu_{M_{t}}$, and the normal of the barrier $\Sigma, \nu_{\Sigma}(F)$, are perpendicular; that is,

$$
\nu_{M_{t}} \cdot \nu_{\Sigma}(F)=0
$$

The boundary condition (3) corresponds to a large-scale view of the fire front, as in a large scale bushfire.

We propose to examine the setting where the solution to (1) exists only for a finite time before developing a singularity. A singularity for a curvature flow is a point where the flow cannot be defined, due to a loss of smoothness. This behaviour can occur, for example when the enclosed volume between the evolving surface and the support surface vanishes and the evolving surfaces contracts to a point. In the context of a fire front, the formation of a singularity would be interpreted as extinguishment of the fire.

Based on established results for curvature flows, we expect to be able to find support surfaces $\Sigma$ for which the flow only exists for a finite time before developing a curvature singularity. This result would guide the construction of barriers customised to a particular fire front that in turn control the behaviour of the fire and most importantly lead to finite time extinction of the fire. We conjecture that the following proposition is true. 
Proposition 1 (Finite time extinction of fire fronts with barriers). For specific initial data $M_{0}$ and atmospheric and topographic conditions $f$, there exists a smooth support surface $\Sigma$ such that the solution $F_{t}$ of (1) under the boundary condition (2) or (3) exists for a finite time $T<\infty$ and shrinks as $t \rightarrow T$ to a point on $\Sigma$.

The proposition states that for a given initial fire front $M_{0}$ we can always construct a barrier for the flow such that the fire would be extinguished in finite time. One could also compute in certain cases the exact time of existence by evaluating the rate of convergence to the singularity point. We envisage that the analysis behind this result could be used to develop a numerical simulator capable of predicting the behaviour of a given fire and in turn designing an appropriate barrier. The data obtained would also provide valuable information for understanding the fire and optimising firefighting techniques.

\section{Finite TIME EXTINCTION IN SPECIAL CASES}

There are already theoretical results in the literature related to simpler cases of our model. The proposition above is the analog of the curvature singularity behaviour proved by Stahl (1996), in the restricted case of mean curvature flow without a forcing term and with a spherical or planar barrier. The interpretation of a result of Stahl's relevant to our setting is

Theorem 1. Let $f \equiv 0$ and $\Sigma$ be a sphere of radius $R$ in $\mathbb{R}^{3}$, or let $\Sigma$ be a plane in $\mathbb{R}^{3}$. If the initial surface $M_{0}$ is positively curved everywhere in the sense of the eigenvalues of its second fundamental form, then the solution of the Neumann boundary problem (1) with boundary condition (3) exists only for a finite time. The solution contracts to a point on $\Sigma$ at the final time.

The Theorem states that initial convex surfaces within a support surface that is spherical or planar, will develop a curvature singularity in finite time. The proof is based on preservation of convexity, various curvature bounds from above and below, an argument controlling the deviation of curvatures from each other (first used for mean curvature flow but widely applied), and a theorem of Tso, again used for a large class of flow with a source term (McCoy, 2005). When adapted to the setting of a flow with free boundary, these results combine to show that the volume enclosed between the evolving surfaces and the barrier approaches zero.

Following previous mentioned work, the first author has proved that finite time extinction also applies to a large class of initial surfaces for the case of rotationally symmetric graphs (Wheeler (nee Vulcanov), 2013). Depending on the initial surface and the conditions imposed on $\Sigma$, finite time extinction can be shown using comparison principle techniques.

Theorem 2. Let $f=0$ and suppose $\Sigma$ given by $\left(x_{1}, x_{2}, \omega_{\Sigma}\left(\sqrt{x_{1}^{2}+x_{2}^{2}}\right)\right)$ is a rotationally symmetric barrier defined on $\left[0, r_{\Sigma}\right]$ such that $\omega_{\Sigma}\left(r_{\Sigma}\right)=0$ For $y=\sqrt{x_{1}^{2}+x_{2}^{2}}$ let $\Sigma$ satisfy the conditions $\omega_{\Sigma}(y) \frac{d \omega_{\Sigma}}{d y}(y)<0$, for $y \in\left(0, r_{\Sigma}\right)$ and $\frac{d \omega_{\Sigma}}{d y}(0)=0$ (which correspond to a negative, decreasing slope from a global maximum point at $y=0$ if $\omega_{\Sigma}$ is a positive function and to a positive, increasing slope from a global minimum at $y=0$ for the negative functions $\left.\omega_{\Sigma}\right)$. Let $M_{t}$ be given by the rotation of a graph function $\omega$ defined on a domain $(r(t), R)$ such that $R \notin\left[0, r_{\Sigma}\right]$ and $r(0) \leq r_{\Sigma}<R$. Suppose also that there exists a $y \in[r(0), R]$ such that $\left|\omega_{0}\right|(y)>\left|\omega_{\Sigma}(0)\right|$. If there exists a shrinking torus in the region of $\mathbb{R}^{3}$ defined as $\left\{\left(x_{1}, x_{2}, x_{3}\right):\left|\omega_{\Sigma}(0)\right|<\right.$ $\left.\left|x_{3}\right|<\left|\omega_{0}(y)\right|\right\}$ then the solution for the problem (1) under the boundary condition (3) exists for only a finite time $T<\infty$ and the graphs $\omega(\cdot, t)$ develop a curvature singularity at $y=0$ as $t \rightarrow T$.

Rotationally symmetric surfaces can be used as comparison surfaces for the more general flow to show that even without the rotational symmetry some general surfaces exist under the flow (1) only for a finite time. A comparison principle between initially disjoint surfaces shows that under the flow they remain disjoint. Constructing the rotationally symmetric comparison surfaces such that they enclose the initial front will suffice to obtain a similar singular behaviour for the evolving surface.

A similar method should apply to the more general flow (1). The source term may be dealt with by applying similar techniques to those used by other authors (for example, see McCoy (2005), Li et al. (2009) and the references therein). Given the source term does not depend upon curvature; that is, it is a lower-order term, the requirements on it to facilitate important intermediate estimates using the maximum principle for parabolic partial differential equations are generally mild. If the magnitude of the source term is large relative to the curvature term then the flow behaviour becomes more like that of a lower-order flow. We expect that when the source term is included the result will hold true for a much larger variety of support surfaces $\Sigma$ than only the spheres and planes discussed by Stahl (1996) or the rotationally symmetric case discussed by Wheeler (nee Vulcanov) (2013). 
We note that by construction the problem has two boundaries: the ground; and the maximal height of the fuel. The ground can be viewed (at least in flat terrain) as a planar barrier to which the ignition surfaces is tangential, in which case we can take the boundary condition (2). Similarly, we can view the maximum fuel height as a planar boundary, for which the boundary conditions (3) apply (see Fig. 2). The ignition surface evolves between two barriers, above and below. We conjecture that based on the initial configuration of the fire front and the fuel type we will be able to analytically prove that certain barriers, to be constructed in between the upper and lower barriers, would force the front quickly into extinction.

Examples. Let us consider first the case when the ignition surface moves between two planes at zero and a given constant height, with homogeneous fuel, atmospheric and topographic conditions. Based on previous results on curvature flow we expect that a barrier constructed as a back-burned planar or sphere-like region would force the fire front into extinction. This corresponds to a systematic back-burning of the fuel such that the unburned region ahead of the fire front has constant curvature. For a more complex configuration in which the lower barrier is already curved, such as when the front would move up or down a hill, the region to be constructed through back-burning could be a combination of appropriate surfaces.

\section{VAlidating The MOdel}

The extra dimensionality accommodated by the proposed model poses some challenges when it comes to model validation. Fire spread is often viewed as a surface phenomenon and thus rate of spread data often only provides information on the surface rate of spread. Observation of spread rates that generally differ across different vegetation strata (e.g. surface, shrub layer, crown fire) is inherently more difficult. One possibility might be to use infrared/thermal data that permits a more holistic determination of the fire front, or of sections of surfaces of specified temperatures. This would allow for a more three-dimensional appraisal of how the performance of the proposed model accords with observed fire spread characteristics.

Other options include evaluation of the model at different heights, so that the rate of spread of the fire through elevated fuels (e.g. the forest canopy) could be compared with experimental fire data that is particularly relevant to crown fire progression (Cruz and Alexander, 2010, and the references therein). Recent data on fire progression through shrub-like vegetation (Cruz et al., 2013) may also be of use in testing and developing the proposed model.

At larger scales, satellite data that carries information on the degree of crown scorch could also be used to compare with predictions obtainable from the proposed model. So while the more mathematically sophisticated modelling framework does provide some challenges in finding means to validate the model, there are a number of promising options. The model itself will also likely suggest additional measures and metrics that might be considered as part of experimental fire programs to form a broader basis for model evaluation more generally.

\section{Conclusions}

The proposed model has the potential to improve the way a fire front is modelled through the following new additions. The extra dimensionality allows more accurate modelling of the ignition isosurface for a fuel bed with variable height or for discontinuous fuel beds. The nonlinear speed is more consistent with experimental data, which confirms that initial corners and irregularities of the front will smoothen out over relatively short timeframes. This latter feature, in particular, could be beneficial for firefighter safety as it will better allow for prediction of dynamic fire spread, types of which have been associated with firefighter fatalities in the past (Viegas, 2009).

Moreover, the proposed modelling approach naturally allows the novelty of barriers as part of the mathematical framework, and these can be used to force the front into extinction. Previous work suggests that the proposed approach is feasible and that it can be seen as an initial step into the analytical design of barriers which could be used in firefighting techniques and suppression strategies.

\section{ACKNOWLEDGEMENT}

V.-M. Wheeler, J.A. McCoy and G.E. Wheeler are supported by Discovery Grant DP120100097 of the Australian Research Council (ARC). They are also grateful for the support of the Institute for Mathematics and its Applications at the University of Wollongong. J.J. Sharples is supported by the ARC Discovery Indigenous Award IN130100038 and by a UNSW Canberra Silver Star Award. 
V.-M. Wheeler, J. A. McCoy, G. E. Wheeler, J. J. Sharples, Curvature flows and barriers in fire modelling

\section{REFERENCES}

Almeida, L., A. Chambolle, and M. Novaga (2012). Mean curvature flow with obstacles. Annales de l'Institut Henri Poincarée 29, 667-681.

Anderson, D. H., E. A. Catchpole, N. J. DeMaestre, and T. Parkes (1982). Modelling the spread of grass fires. J. Austral. Math. Soc Ser. B 23, 451-466.

Andrews, B. H. (2001). Volume-preserving anisotropic mean curvature flow. Indiana Univ. Math. J. 50(2), 783-827.

Andrews, B. H., J. A. McCoy, and Y. Zheng (2013). Contracting convex hypersurfaces by curvature. Calc. Var. 47, 611-665.

Bressan, A. and T. Wang (2012). Global optimality conditions for a dynamic blocking problem. ESAIM Control Optim. Calc. Var 18, 124-156.

Cruz, M. G. and M. E. Alexander (2010). Assessing crown fire potential in coniferous forests of western North America: a critique of current approaches and recent simulation studies. International Journal of Wildland Fire 19, 377-298.

Cruz, M. G., W. L. McCaw, W. A. Anderson, and J. S. Gould (2013). Fire behaviour modelling in semi-arid malle-heath shrublands of southern Australia. Environmental Modelling and Software 40, 21-34.

Huisken, G. (1984). Flow by mean curvature of convex surfaces into spheres. J. Differential Geom. 20(1), 237-266.

Li, G., L. Yu, and C. Wu (2009). Curvature flow with a general forcing term in euclidean spaces. Journal of Mathematical Analysis and Applications 353(2), 508-520.

Mallet, V., D. Keyes, and F. Fendell (2009). Modeling wildland fire propagation with level set methods. Computers \& Mathematics with Applications 57(7), 1089-1101.

McCoy, J. A. (2005). Mixed volume preserving curvature flows. Calc. Var. 24(2), 131-154.

Mullins, W. W. (1956). Two-dimensional motion of idealized grain boundaries. J. Appl. Phys. 27, 900-904.

Osher, S. and J. A. Sethian (1988). Fronts propagating with curvature-dependent speed: algorithms based on hamilton-jacobi formulations. J. Comp. Phys. 79(1), 12-49.

Perry, G. L. W. (1998). Current approaches to modelling the spread of wildland fire: a review. Progress in Physical Geography 22(2), 222-245.

Roberts, S. (1993). A line element algorithm for curve flow problems in the plane. J. Austral. Math. Soc. 35, 244-261.

Rothermel, R. (1972). A mathematical model for predicting fire spread in wildland fuels. USDA Forest Service Research Paper INT-115.

Sapiro, G. and A. Tannenbaum (1994). On affine plane curve evolution. J. Funct. Anal. 119, 79-120.

Séro-Guillaume, O., S. Ramezani, J. Margerit, and D. Calogine (2007). On large scale forest fires propagation models. International Journal of Thermal Sciences 47, 680-694.

Stahl, A. (1996). Regularity estimates for solutions to the mean curvature flow with a neumann boundary condition. Calc. Var. 4(4), 385-407.

Viegas, D. X. (2009). Recent Forest Fire Related Accidents in Europe. Publications Office of the European Union.

Weber, R. O. and H. S. Sidhu (2006). A dynamical systems model for fireline growth with suppression. ANZIAM J. 47, 462-474.

Wheeler (nee Vulcanov), V.-M. (2012). Mean curvature flow of entire graphs in a half-space with a free boundary. J. Reine Angew. Math., DOI: 10.1515/crelle-2012-0028.

Wheeler (nee Vulcanov), V.-M. (2013). Non-parametric radially symmetric mean curvature flow with a free boundary. Math. Z.., DOI: 10.1007/s00209-013-1200-7. 